\title{
The Unruh effect for mixing neutrinos
}

Article $\cdot$ March 2018

DOl: 10.1103/PhysRevD.97.105022

CITATION

5 authors, including:

Sers Stephen A. Fulling

161 PUBLICATIONS 6,046 CITATIONS

SEE PROFILE

A George E. A. Matsas

São Paulo State University

88 PUBLICATIONS 1,699 CITATIONS

SEE PROFILE

Some of the authors of this publication are also working on these related projects:

Casimir Interactions View project

Project Boundary-energy anomaly View project
READS

88

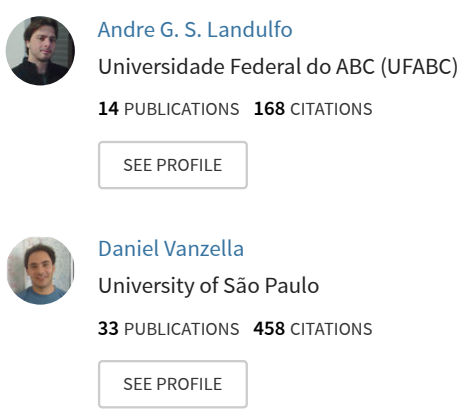




\title{
The Unruh effect for mixing neutrinos
}

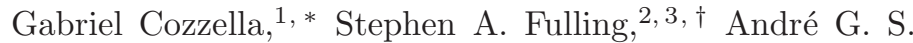

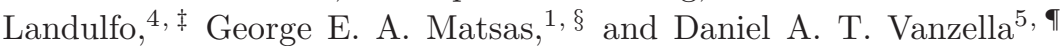 \\ ${ }^{1}$ Instituto de Física Teórica, Universidade Estadual Paulista, \\ Rua Dr. Bento Teobaldo Ferraz, 271, 01140-070, São Paulo, São Paulo, Brazil \\ ${ }^{2}$ Department of Physics, Texas A\&M University, College Station, Texas, USA \\ ${ }^{3}$ Department of Mathematics, Texas A $6 M$ University, College Station, Texas, 7r843-3368, USA \\ ${ }^{4}$ Centro de Ciências Naturais e Humanas, Universidade Federal do ABC, \\ Avenida dos Estados, 5001, 09210-580, Santo André, São Paulo, Brazil \\ ${ }^{5}$ Instituto de Física de São Carlos, Universidade de São Paulo, \\ Caixa Postal 369, 13560-970, São Carlos, São Paulo, Brazil
}

(Dated: March 20, 2018)

\begin{abstract}
Recently, the inverse $\beta$-decay rate calculated with respect to uniformly accelerated observers (experiencing the Unruh thermal bath) was revisited. Concerns have been raised regarding the compatibility of inertial and accelerated observers' results when neutrino mixing is taken into account. Here we show that these concerns are unfounded by discussing the properties of the Unruh thermal bath with mixing neutrinos and explicitly calculating the decay rates according to both sets of observers and confirming that they are in agreement. The Unruh effect is perfectly valid for mixing neutrinos.
\end{abstract}

\section{INTRODUCTION}

The Unruh effect was initially derived assuming free quantum fields [1]. Later, it was shown to be valid also for interacting ones [2 [5]. Surprisingly, perhaps, only recently the Unruh effect has been discussed in the context of mixing neutrinos, with disturbing conclusions being drawn. In Ref. [6], it is claimed that the inverse $\beta$-decay rate for an accelerated proton as calculated with respect to inertial and uniformly accelerated observers (experiencing the Unruh thermal bath) would disagree with respect to each other when taking into account the existence of multiple families of mixing neutrinos. We claim that this is impossible because calculations of observables realized in different frames must necessarily agree, in conformity with the general covariance of quantum field theory. Thus, either the Unruh effect is wrong (contradicting several previous results [7], including what we consider to be a virtual observation of it [8]) or some mistake was made in the previously mentioned calculation. The purpose of this paper is to discuss the Unruh thermal bath for mixing neutrino fields and also revisit the inverse $\beta$ decay for accelerated protons with the aim to show that the Unruh effect is perfectly valid in this setting.

The paper is organized as follows. In Sec.[II we discuss the Unruh thermal bath for the case of mixing neutrinos with particular attention to when can we consider flavor particle states as legitimate quantum states and how this reflects in measurements made involving the Unruh ther-

\footnotetext{
*Electronic address: cozzella@ift.unesp.br

${ }^{\dagger}$ Electronic address: fulling@math.tamu.edu

‡Electronic address: andre.landulfo@ufabc.edu.br

$\S$ Electronic address: matsas@ift.unesp.br

IElectronic address: vanzella@ifsc.usp.br
}

mal bath. In Section ஹw we set the stage for calculating the inverse $\beta$-decay rate for accelerated protons. Section IV] concerns the calculation of the inverse $\beta$-decay rate from the inertial point of view. In Sec. $\mathrm{V}$, we calculate independently the inverse $\beta$-decay rate from the point of view of uniformly accelerated observers and show that the result is in full agreement with the one previously obtained in Sec. IV] Our closing remarks appear in Sec. VI

Throughout this work we use $(+,-,-,-)$ signature for the Minkowski metric, $\eta_{\mu \nu}$, and natural units, $\hbar=c=$ $k_{\mathrm{B}}=1$, unless stated otherwise. The same conventions as in Ref. 9] are followed for the Dirac matrices and normal modes.

\section{THE UNRUH THERMAL BATH WITH MIXING NEUTRINOS}

The Unruh effect says that while inertial observers see the Minkowski vacuum state as a no-particle state, uniformly accelerated observers (restricted to the Rindler wedges) see it as a thermal state with a temperature proportional to their proper acceleration. This statement applies both to the vacuum state of free and interacting fields. Particularly, it applies to massive neutrino fields, $\hat{\nu}_{i}, i \in\{1,2,3\}$. Hence, before dealing with neutrino mixing, let us begin by setting our notation and briefly reviewing some relevant features of the Unruh thermal bath.

Consider a massive fermionic field $\hat{\psi}$ satisfying the Dirac equation with mass $m$. Inertial observers evolving in Minkowski spacetime covered with usual Cartesian coordinates $\{t, x, y, z\}$ expand it as

$$
\hat{\psi}=\sum_{\sigma= \pm} \int d^{3} k\left(\hat{a}_{\vec{k}, \sigma} u_{\vec{k}, \sigma}^{+\omega}+\hat{b}_{\vec{k}, \sigma}^{\dagger} u_{\vec{k},-\sigma}^{-\omega}\right),
$$


where $\vec{k}=\left(k^{x}, k^{y}, k^{z}\right)$ and $\sigma$ are the fermion's momentum and spin, respectively, and $\omega^{2}=|\vec{k}|^{2}+m^{2}$. The positive $(+)$ and negative $(-)$ frequency modes (with respect to $\left.\partial_{t}\right) u_{\vec{k}, \sigma}^{ \pm \omega}$ are given by

$$
u_{\vec{k}, \sigma}^{ \pm \omega}=\frac{e^{\mp i k_{\mu} x^{\mu}}}{(2 \pi)^{3 / 2}} v_{\sigma}^{ \pm \omega}(\vec{k})
$$

where $k^{\mu}=(\omega, \vec{k}), x^{\mu}=(t, x, y, z)$,

$$
v_{\sigma}^{ \pm \omega}(\vec{k})=\frac{\left(k_{\mu} \gamma^{\mu} \pm m \mathbb{I}\right)}{\sqrt{[2 \omega(\omega \pm m)]}} \hat{v}_{\sigma},
$$

$\gamma^{\mu}=\left(\gamma^{0}, \gamma^{1}, \gamma^{2}, \gamma^{3}\right)$ being the Dirac matrices and

$$
\hat{v}_{+}=\left[\begin{array}{l}
1 \\
0 \\
0 \\
0
\end{array}\right], \quad \hat{v}_{-}=\left[\begin{array}{l}
0 \\
1 \\
0 \\
0
\end{array}\right] .
$$

The modes are orthonormalized according to the inner product,

$$
(\psi, \phi) \equiv \int d \Sigma_{\mu} \bar{\psi} \gamma^{\mu} \phi
$$

where $\bar{\psi}=\psi^{\dagger} \gamma^{0}$. The fermionic annihilation and antifermionic creation operators, $\hat{a}_{\vec{k}, \sigma}$ and $\hat{b}_{\vec{k}, \sigma}^{\dagger}$, satisfy the usual anti-commutation relations,

$$
\begin{aligned}
& \left\{\hat{a}_{\vec{k}, \sigma}, \hat{a}_{\vec{k}^{\prime}, \sigma^{\prime}}^{\dagger}\right\}=\delta^{3}\left(\vec{k}-\vec{k}^{\prime}\right) \delta_{\sigma, \sigma^{\prime}}, \\
& \left\{\hat{b}_{\vec{k}, \sigma}, \hat{b}_{\vec{k}^{\prime}, \sigma^{\prime}}^{\dagger}\right\}=\delta^{3}\left(\vec{k}-\vec{k}^{\prime}\right) \delta_{\sigma, \sigma^{\prime}},
\end{aligned}
$$

with all others vanishing. We define the Minkowski vacuum state, $\left|0_{\mathrm{M}}\right\rangle$, as the state annihilated by all annihilation operators, i.e.,

$$
a_{\vec{k}, \sigma}\left|0_{\mathrm{M}}\right\rangle=b_{\vec{k}, \sigma}\left|0_{\mathrm{M}}\right\rangle=0, \quad \forall \vec{k}, \sigma .
$$

Rindler observers, accelerating uniformly in the $z$ direction with proper acceleration $a$, on the other hand, quantize the field using a different set of normal modes more appropriate to them. In order to later also discuss the decay of accelerated protons from the point of view of uniformly accelerated observers, it is convenient to cover the right Rindler wedge portion $z>|t|$ of Minkowski spacetime with Rindler coordinates $(v, x, y, u)$, where

$$
\begin{aligned}
& v=\tanh ^{-1}(t / z), \\
& u=\sqrt{z^{2}-t^{2}} .
\end{aligned}
$$

The line element is written as

$$
d s^{2}=u^{2} d v^{2}-d x^{2}-d y^{2}-d u^{2} .
$$

In these coordinates we write $\hat{\psi}$ as

$$
\begin{array}{r}
\hat{\psi}=\sum_{\sigma= \pm} \int_{0}^{\infty} d \varpi \int d^{2} k_{\perp}\left[\hat{c}_{\varpi, \vec{k}_{\perp}, \sigma} g_{\vec{k}_{\perp}, \sigma}^{+\varpi}\right. \\
\left.+\hat{d}_{\varpi, \vec{k}_{\perp}, \sigma} g_{\vec{k}_{\perp},-\sigma}^{-\varpi}\right],
\end{array}
$$

where $\varpi$ stands for the Rindler frequency, and $\vec{k}_{\perp} \equiv$ $\left(k^{x}, k^{y}\right)$ labels the transverse momentum quantum number. The positive and negative modes (with respect to $\partial_{v}$ ), orthonormalized according to Eq. (5), can be cast as

$$
g_{\vec{k}_{\perp}, \sigma}^{ \pm \varpi}=\frac{e^{\mp i \varpi v / a+i \vec{k}_{\perp} \cdot \vec{x}_{\perp}}}{(2 \pi)^{3 / 2}} h_{\sigma}\left( \pm \varpi, \vec{k}_{\perp}\right),
$$

where $\vec{x}_{\perp}=(x, y)$ and

$$
\begin{array}{r}
h_{\sigma}\left( \pm \varpi, \vec{k}_{\perp}\right)=\left[\frac{\cosh (\varpi \pi / a)}{\pi a l}\right]^{1 / 2} \\
\times \gamma^{0}\left[\left(\vec{k}_{\perp} \cdot \vec{\gamma}_{\perp}+m \mathbb{I}\right) K_{ \pm i \varpi / a+1 / 2}(l u)\right. \\
\left.+i l \gamma^{3} K_{ \pm i \varpi / a-1 / 2}(l u)\right] \hat{h}_{\sigma},
\end{array}
$$

with

$$
\hat{h}_{+}=\left[\begin{array}{l}
1 \\
0 \\
1 \\
0
\end{array}\right], \quad \hat{h}_{-}=\left[\begin{array}{c}
0 \\
1 \\
0 \\
-1
\end{array}\right]
$$

$l^{2}=\left|\vec{k}_{\perp}\right|^{2}+m^{2}$ and $\vec{\gamma}_{\perp} \equiv\left(\gamma^{1}, \gamma^{2}\right) . \hat{c}_{\varpi, \vec{k}_{\perp}, \sigma}$ and $\hat{d}_{\varpi, \vec{k}_{\perp}, \sigma}^{\dagger}$ are the Rindler fermionic annihilation and anti-fermionic creation operators also satisfying relations (6)-(7) with appropriate quantum numbers (with all others also vanishing). The associated vacuum, $\left|0_{\mathrm{R}}\right\rangle$, is such that

$$
\hat{c}_{\varpi, \vec{k}_{\perp}, \sigma}\left|0_{\mathrm{R}}\right\rangle=\hat{d}_{\varpi, \vec{k}_{\perp}, \sigma}\left|0_{\mathrm{R}}\right\rangle=0, \quad \forall \varpi, \vec{k}_{\perp}, \sigma .
$$

By relating both set of normal modes via a Bogulubov transformation [7], the state seen by Rindler observers restricted to the right Rindler wedge can be written as

$$
\begin{aligned}
\hat{\rho}_{\beta_{U}} & =\bigotimes_{\varpi, \vec{k}_{\perp}, \sigma} Z_{\varpi} \sum_{n=0}^{1} \exp (-2 \pi n \varpi / a) \\
& \times\left|n ; \varpi, \vec{k}_{\perp}, \sigma\right\rangle\left\langle n ; \varpi, \vec{k}_{\perp}, \sigma\right|,
\end{aligned}
$$

where $Z_{\varpi}^{-1}=1+\exp (-2 \pi \varpi / a) . \hat{\rho}_{\beta_{U}}$ is a thermal state at inverse temperature $\beta_{U}=2 \pi / a$, clearly showing the Unruh effect.

Taking our fermionic fields to be the massive neutrino fields $\hat{\nu}_{i}$, we see from Eq. (17) that the mean flux of neutrinos with a well-defined mass $m_{i}$, energy $\varpi$, transverse momentum $\vec{k}_{\perp}$, and spin $\sigma$ seen by a Rindler observer is given by

$$
\begin{aligned}
\bar{n}\left(\varpi, \vec{k}_{\perp}, \sigma\right) & \equiv \frac{d}{d \tau} \frac{d^{2}}{d^{2} x_{\perp}} \lim _{\substack{\varpi^{\prime} \rightarrow \varpi \\
\vec{k}_{\perp}^{\prime} \rightarrow \vec{k}_{\perp}}}\left\langle c_{\varpi^{\prime}, \vec{k}_{\perp}^{\prime}, \sigma}^{c_{\varpi,}} c_{\vec{k}_{i \perp}, \sigma}^{i}\right\rangle_{\hat{\rho}_{\beta_{U}}} \\
& =(2 \pi)^{-3} n_{F}(\varpi),
\end{aligned}
$$

where

$$
n_{F}(\varpi)=\left(1+e^{\beta_{U} \varpi}\right)^{-1},
$$


and $\tau$ is the observer's proper time. As expected, the result is proportional to the Fermi-Dirac factor and only depends on the Rindler energy $\varpi$. In particular, there is no dependency on the neutrino's mass.

This does not mean, however, that detectors sensitive to $\nu_{i}$ with different masses $m_{i}$ carried by a given Rindler observer would react with the Unruh thermal bath in the same way.

To better understand this point, let us define a generalization of the Unruh-deWitt detector for neutrinos, which is constructed with the interaction action

$$
\hat{S}_{D} \equiv \lambda \sum_{i} \int d \tau\left[\hat{\bar{m}}(\tau) \hat{\nu}_{i}\left[x_{D}^{\mu}(\tau)\right]+\text { H. c. }\right],
$$

where $\lambda$ is a (dimensional) constant, $x_{D}^{\mu}(\tau)$ is the detector's trajectory, and

$$
\hat{m}(\tau)=\hat{m}_{0}(\tau)\left(\begin{array}{l}
\hat{\eta} \\
\hat{\xi}
\end{array}\right)
$$

with $\hat{m}_{0}(\tau)$ being a monopole operator such that

$$
\left\langle e\left|\hat{m}_{0}(\tau)\right| g\right\rangle=e^{i \Delta E \tau}\left\langle e\left|\hat{m}_{0}(0)\right| g\right\rangle .
$$

Here, $\Delta E$ is the energy gap between the detector's excited, $|e\rangle$, and unexcited, $|g\rangle$, states of the detector, and $\hat{\eta}$ and $\hat{\xi}$ are arbitrary bi-spinors satisfying the normalization conditions $\hat{\eta}^{\dagger} \hat{\eta}=\hat{\xi}^{\dagger} \hat{\xi}=1$. (See Appendix $\mathrm{A}$ for an application of our detector in the simpler setting of an inertial thermal bath where we comment on the reasonable properties this detector possess).

The worldline of a uniformly accelerated detector with proper acceleration $a$, in the $(v, x, y, u)$ coordinates is taken to be

$$
x_{D}^{\mu}(\tau)=(a \tau, 0,0,1 / a) .
$$

The mean excitation rate per proper time by absorption of a neutrino with mass $m_{i}$ is then

$$
\frac{d P_{\mathrm{exc}, i}}{d \tau}=\frac{d}{d \tau} \sum_{\sigma= \pm} \int_{0}^{\infty} d \varpi \int d^{2} k_{\perp}\left|\mathcal{A}_{\mathrm{exc}}\right|^{2} n_{F}(\varpi),
$$

with

$$
\mathcal{A}_{\mathrm{exc}}=i\left\langle e ; 0_{\mathrm{R}}\left|\hat{S}_{D}\right| g ; \nu_{\varpi, \vec{k}_{\perp}, \sigma}\right\rangle
$$

which gives

$$
\begin{aligned}
\frac{d P_{\mathrm{exc}, i}}{d \tau} & =\Lambda^{2} \int_{0}^{\infty} d \varpi \delta(\varpi-\Delta E) e^{-\pi \varpi_{R} / a} \\
& \times \int d^{2} k_{\perp}\left(l_{i} / a\right)\left|K_{i \varpi_{R} / a+1 / 2}\left(l_{i} / a\right)\right|^{2}
\end{aligned}
$$

where the constant $\Lambda^{2}=|\lambda|^{2}\left|\left\langle e\left|\hat{m}_{0}(0)\right| g\right\rangle\right|^{2} / 2 \pi^{3}$ depends on the detector's specifics and we recall that $l_{i}^{2}=\left|\vec{k}_{\perp}\right|^{2}+$ $m_{i}^{2}$. Note that the detector is only sensitive to particles with Rindler energy $\varpi=\Delta E$.
The excitation rate should also be proportional to the local neutrino density. We can confirm this by extending Ref. [10], which builds on ideas from quantum optics to define a finite-volume particle number operator for finite space volume using the scalar Klein-Gordon product.

In our case, using Eq. (5), we define the neutrino density operator in an infinitesimal volume around the detector as

$$
\begin{aligned}
\hat{n}_{i, D} & \equiv d\left(\nu_{i}^{+}, \nu_{i}^{+}\right) /\left.d u d^{2} x_{\perp}\right|_{x^{\mu}(\tau)=x_{D}^{\mu}(\tau)} \\
& =\left.\bar{\nu}_{i}^{+} \gamma^{0} \nu_{i}^{+}\right|_{x^{\mu}(\tau)=x_{D}^{\mu}(\tau)},
\end{aligned}
$$

where $\nu_{i}^{+}$denotes the positive frequency part of Eq. (12) and Eq. (27) is calculated over a surface $v=$ const. The expectation value of this density operator in the Minkowski vacuum $\left|0_{\mathrm{M}}\right\rangle$ is

$$
\begin{aligned}
\left\langle 0_{\mathrm{M}}\left|\hat{n}_{i, D}\right| 0_{\mathrm{M}}\right\rangle & =\frac{1}{2 \pi^{4}} \int_{0}^{\infty} d \varpi e^{-\pi \varpi_{R} / a} \int d^{2} k_{\perp} \\
& \times\left(l_{i} / a\right)\left|K_{i \varpi_{R} / a+1 / 2}\left(l_{i} / a\right)\right|^{2} .
\end{aligned}
$$

Clearly, Eq. (26) is proportional to Eq. (29) when restricted to particles with $\varpi=\Delta E$, confirming that the excitation rate is proportional to the local neutrino density.

We note from Eq. (26) that Rindler observers will have a harder time detecting more massive neutrinos since they concentrate closer to the horizon. This is in accordance with previous results obtained in the scalar case [10, 11].

Let us consider now the properties of the Unruh thermal bath in terms of flavor neutrinos. It is possible to define phenomenologically flavor states $\left|\nu_{\alpha} ; \vec{k}, \sigma\right\rangle$ and $\left|\nu_{\alpha} ; \varpi, \vec{k}_{\perp}, \sigma\right\rangle$ for inertial and Rindler observers, respectively, in the realm of usual quantum field theory. Their usual form arise as an useful approximation in the regime where, e.g., the involved momenta are larger than the neutrino masses. For more details we point the reader to Refs. [17], 18], 19] and [20] (see also the final paragraph of Sec. III). From the inertial perspective, by looking at Eq. (2), this regime is

$$
|\vec{k}|^{2} \gg m_{i}^{2}, \forall i
$$

while from the Rindler perspective, by looking at Eq. (14) we see that this will be the case wherever we deal with neutrinos that have transverse momentum satisfying

$$
\left|\vec{k}_{\perp}\right|^{2} \gg m_{i}^{2}, \forall i .
$$

The flavor neutrino states are then written as

$$
\begin{aligned}
\left|\nu_{\alpha} ; \vec{k}, \sigma\right\rangle & \equiv \sum_{i} U_{\alpha, i}^{*}\left|\nu_{i} ; \vec{k}, \sigma\right\rangle, \\
\left|\nu_{\alpha} ; \varpi, \vec{k}_{\perp}, \sigma\right\rangle & \equiv \sum_{i} U_{\alpha, i}^{*}\left|\nu_{i} ; \varpi, \vec{k}_{\perp}, \sigma\right\rangle .
\end{aligned}
$$

where $\alpha \in\{e, \mu, \tau\}$ labels the leptonic flavor and $U_{\alpha, i}$ is the PMNS matrix [13]. 
In this regime, we can ask, for instance, what is the excitation rate for an $\alpha$-flavor neutrino. From Eq. (33) we see that the probability of a $\nu_{i}$ reaching the detector to collapse as a $\nu_{\alpha}$ is $\left|U_{\alpha, i}\right|^{2}$, therefore

$$
\begin{aligned}
\left.\frac{d P_{\mathrm{exc}, \alpha}}{d \tau}\right|_{\left|\vec{k}_{\perp}\right|^{2} \gg m_{i}^{2}} & =\left.\sum_{i}\left|U_{\alpha, i}\right|^{2} \frac{d P_{\mathrm{exc}, i}}{d \tau}\right|_{\left|\vec{k}_{\perp}\right|^{2} \gg m_{i}^{2}} \\
& \left.\approx \frac{d P_{\mathrm{exc}, i}}{d \tau}\right|_{m_{i}=0}
\end{aligned}
$$

due to the unitarity of the PMNS matrix.

We conclude that the predicted behavior of flavor neutrinos (when well-defined) is in no way deviant from what it would be expected from the thermality of the Unruh bath. We proceed now to discuss the inverse $\beta$ decay for accelerated protons with neutrino mixing from the inertial and accelerated observers' view and show that it is also consistent with the Unruh effect.

\section{SEMI-CLASSICAL INVERSE $\beta$ DECAY WITH NEUTRINO MIXING}

For the sake of our purposes, we adopt the same approach as [6, 12], where the proton, $|p\rangle$, and neutron, $|n\rangle$, are seen as unexcited and excited states of a two-level system with the corresponding Hamiltonian $\hat{H}$ satisfying

$$
\begin{aligned}
\hat{H}|p\rangle & =m_{p}|p\rangle, \\
\hat{H}|n\rangle & =m_{n}|n\rangle,
\end{aligned}
$$

where $m_{p(n)}$ is the proton (neutron) mass. The protonneutron system is assumed to have a well-prescribed spacetime trajectory described by the semi-classical current:

$$
\hat{j}^{\mu}=\frac{\hat{q}(\tau)}{\sqrt{-g} u^{0}} u^{\mu}(\tau) \delta^{3}\left(\vec{x}-\vec{x}_{0}(\tau)\right)
$$

where $g=\operatorname{det}\left(\eta_{\mu \nu}\right), u^{\mu}(\tau)$ is the four velocity of the linearly accelerated proton-neutron system with proper time $\tau$, proper acceleration $a=$ const, and $\vec{x}_{0}(\tau)$ is its spatial trajectory. The monopole operator $\hat{q}(\tau)$ is defined via the Hamiltonian by

$$
\hat{q}(\tau)=e^{i \hat{H} \tau} \hat{q}(0) e^{-i \hat{H} \tau}
$$

where the Fermi constant, $G_{F}$, will be given by $G_{F} \equiv$ $|\langle n|\hat{q}(0)| p\rangle|$. The leptonic fields, in turn, will be treated as quantum fields.

The effective weak interaction action considered here is

$$
\begin{aligned}
& \hat{S}_{I}=\int d^{4} x \sqrt{-g}\left(\sum_{\alpha} \hat{\bar{\nu}}_{\alpha} \gamma^{\mu} \hat{P}_{L} \hat{l}_{\alpha} \hat{j}_{\mu}+\text { H. c. }\right) \\
& =\int d^{4} x \sqrt{-g}\left(\sum_{\alpha, i} U_{\alpha, i}^{*} \hat{\bar{\nu}}_{i} \gamma^{\mu} \hat{P}_{L} \hat{l}_{\alpha} \hat{j}_{\mu}+\text { H. c. }\right),
\end{aligned}
$$

where

$$
\hat{P}_{L} \equiv \frac{\left(\mathbb{I}-\gamma^{5}\right)}{\sqrt{2}},
$$

$\gamma^{5} \equiv i \gamma^{0} \gamma^{1} \gamma^{2} \gamma^{3}$, and we recall that $\hat{j}^{\mu}$ is given by Eq. (37), $\alpha \in\{e, \mu, \tau\}$ labels the leptonic flavor,

$$
\hat{\nu}_{\alpha} \equiv \sum_{i} U_{\alpha, i} \hat{\nu}_{i}
$$

and $\hat{l}_{\alpha}$ stand for all electrically charged leptonic fields, $\left\{e^{-}, \mu^{-}, \tau^{-}\right\}$.

We stress here that $\hat{\nu}_{\alpha}$ stands only as a shorthand notation for the particular combination of massive neutrino fields given by Eq. (41). As mentioned previously in Sec. III. flavor states can only be defined phenomenologically. Attempts at canonically quantizing the $\hat{\nu}_{\alpha}$ fields in terms of positive (and negative) frequency modes give annihilation (and creation) operators whose physical meaning is unclear [22], precluding us from defining particle states as states resulting from the action of creation operators acting on a vacuum and constructing the associated Fock space 23]. For this reason, we focus only on states associated with the $\hat{\nu}_{i}$ fields from now on.

\section{INERTIAL CALCULATION}

The inverse $\beta$-decay process, as seen by Minkowski observers, can be generically cast in the form

$$
p \rightarrow n \bar{l}_{\alpha} \nu_{i}
$$

where $l_{\alpha}=\left\{e^{-}, \mu^{-}, \tau^{-}\right\}$and $\nu_{i}=\left\{\nu_{1}, \nu_{2}, \nu_{3}\right\}$. The transition amplitude associated with Eq. (42) is

$$
\mathcal{A}_{\alpha, i}^{I}=i\left\langle n\left|\otimes\left\langle\bar{l}_{\alpha} \nu_{i}\left|\hat{S}_{I}\right| 0_{\mathrm{M}}\right\rangle \otimes\right| p\right\rangle,
$$

where the charged leptons $l_{\alpha}$ and neutrinos $\nu_{i}$ have quantum numbers $\sigma_{\alpha(i)} \in\{+,-\}$ and $\vec{k}_{\alpha(i)}=$ $\left(k_{\alpha(i)}^{x}, k_{\alpha(i)}^{y}, k_{\alpha(i)}^{z}\right)$, and $\hat{S}_{I}$ is given in Eq. (39).

In usual inertial coordinates, $x^{\mu}=(t, x, y, z)$, current (37) is written as

$$
\hat{j}^{\mu}=\frac{\hat{q}(\tau)}{a z} u^{\mu}(\tau) \delta(x) \delta(y) \delta\left(z-\sqrt{t^{2}-a^{-2}}\right),
$$

where $u^{\mu}=(a z(\tau), 0,0, a t(\tau))$ with $t=a^{-1} \sinh (a \tau)$ and $z=a^{-1} \cosh (a \tau)$.

The differential decay probability per momentumspace volume $d V_{k}=d^{3} k_{\alpha} d^{3} k_{i}$ is given by

$$
\frac{d P^{p \rightarrow n \bar{l}_{\alpha} \nu_{i}}}{d V_{k}}=\sum_{\sigma_{\alpha}, \sigma_{i}}\left|\mathcal{A}_{\alpha, i}^{I}\right|^{2},
$$

allowing us to define the decay rate per momentum-space volume as

$$
\frac{d \Gamma^{p \rightarrow n \bar{l}_{\alpha} \nu_{i}}}{d V_{k}}=\frac{1}{\Delta \tau} \frac{d P^{p \rightarrow n \bar{l}_{\alpha} \nu_{i}}}{d V_{k}},
$$


where $\Delta \tau$ is the total proper time along the trajectory of the proton-neutron system. Inserting Eqs. (39), (44), and (1) into Eq. (43), we write Eq. (46) as

$$
\begin{aligned}
& \frac{d \Gamma{ }^{p \rightarrow n \bar{l}_{\alpha} \nu_{i}}}{d V_{k}}=\frac{2 G_{F}^{2}\left|U_{\alpha, i}\right|^{2}}{(2 \pi)^{6}} \\
\times & \int_{-\infty}^{\infty} d \xi \exp \left(2 i\left[\Delta m \xi+a^{-1}\left(\omega_{\alpha} \omega_{i}\right) \sinh (a \xi)\right]\right) \\
\times & \frac{1}{\omega_{\alpha} \omega_{i}}\left[k_{i}^{z} k_{\alpha}^{z}+\omega_{i} \omega_{\alpha}+F\left(k_{i, \alpha}^{x}, k_{i, \alpha}^{y}\right)\right],
\end{aligned}
$$

where $\Delta m \equiv m_{n}-m_{p}$, we have made an inverse boost in the $z$-direction [to factor out the proper time integral implicitly contained in Eq. (45)], and $F\left(k_{i, \alpha}^{x}, k_{i, \alpha}^{y}\right)$ is an odd function of its arguments whose form is not important here since it will not contribute to the decay rate when integrated over $d V_{k}$.

By integrating over momenta, we obtain the total decay rate

$$
\begin{aligned}
\Gamma^{p \rightarrow n \bar{l}_{\alpha} \nu_{i}} & =\frac{G_{F}^{2}\left|U_{\alpha, i}\right|^{2}}{\pi^{4} a} e^{\frac{-\pi \Delta m}{a}} \int_{0}^{\infty} d k_{\alpha} k_{\alpha}^{2} \int_{0}^{\infty} d k_{i} k_{i}^{2} \\
& \times K_{2 i \Delta m / a}\left(2\left(\omega_{\alpha}+\omega_{i}\right) / a\right)
\end{aligned}
$$

where $k_{\alpha(i)} \equiv\left|\vec{k}_{\alpha(i)}\right|$. Now, by using the same complex integration procedure employed in Ref. [9] we can rewrite the expression above as a double integral over the complex plane, i.e.,

$$
\begin{aligned}
& \Gamma^{p \rightarrow n \bar{l}_{\alpha} \nu_{i}}=\frac{G_{F}^{2} a^{5}\left|U_{\alpha, i}\right|^{2}}{32 \pi^{7 / 2}} e^{-\pi \Delta m / a} \\
\times & \int_{C_{t}} \frac{d t}{2 \pi i} \int_{C_{s}} \frac{d s}{2 \pi i}|\Gamma(3-s-t+i \Delta m / a)|^{2} \\
\times & \frac{\Gamma(-s) \Gamma(-t) \Gamma(2-t) \Gamma(2-s)}{\Gamma(3-s-t) \Gamma(7 / 2-s-t)}\left[\frac{m_{\alpha}}{a}\right]^{2 t}\left[\frac{m_{i}}{a}\right]^{2 s},(49)
\end{aligned}
$$

where $C_{s}$ and $C_{t}$ are integration contours containing all poles of the $\Gamma$ functions both in the $s$ and $t$ planes.

Although apparently unwieldy, this expression for the decay rate is convenient for our purposes of analytically confirming the equality between Eq. (49) and the analogous result obtained with respect to Rindler observers, to which we proceed now.

\section{RINDLER CALCULATION}

Uniformly accelerated observers see the single inverse $\beta$-decay process considered by inertial observers, Eq. (42), as a set of three processes, namely
1. $p+l_{\alpha} \rightarrow n+\nu_{i}$,
2. $p+\bar{\nu}_{i} \rightarrow n+\bar{l}_{\alpha}$
3. $p+l_{\alpha}+\bar{\nu}_{i} \rightarrow n$

i.e, protons lying at rest with the Rindler observers would decay into neutrons by the absorption (and possible emission) of leptons from (to) the Unruh thermal bath.

In the $(v, x, y, u)$ coordinate system, current (37) is expressed as

$$
\hat{j}^{\mu}=q u^{\mu} \delta(x) \delta(y) \delta\left(u-a^{-1}\right)
$$

with $u^{\mu}=(a, 0,0,0)$.

To obtain the total decay rate, we must sum incoherently processes (1)-(3). Let us outline now the procedure to calculate the decay rate specifically for process (1), since processes (2) and (3) will be similar. Firstly, we calculate the interaction amplitude by using Eqs. (12) and (39),

$$
\begin{aligned}
\mathcal{A}_{\alpha, i}^{R,(1)} & =i\left\langle n\left|\otimes\left\langle\nu_{i}\left|\hat{S}_{I}\right| l_{\alpha}\right\rangle \otimes\right| p\right\rangle \\
& =\frac{i G_{F}}{(2 \pi)^{2} \sqrt{2}} U_{\alpha, i}^{*} \delta\left(\varpi_{\alpha}-\varpi_{i}-\Delta m\right) \\
& \times\left[\bar{g}_{\vec{k}_{i \perp}, \varpi_{i}} \gamma^{0}\left(\mathbb{I}-\gamma^{5}\right) g_{\vec{k}_{\alpha \perp}, \sigma_{\alpha}}^{+\varpi_{\alpha}}\right]
\end{aligned}
$$

where the neutrino $\nu_{i}$ has quantum numbers $\left(\sigma_{i}, \varpi_{i}, \vec{k}_{i \perp}\right)$ and the charged lepton has quantum numbers $\left(\sigma_{\alpha}, \varpi_{\alpha}, \vec{k}_{\alpha \perp}\right)$ and we recall that $\bar{g}=g^{\dagger} \gamma^{0}$. We square it to obtain the differential probability of decay per Rindler momentum-space volume $d V_{k, R}=d \varpi_{\alpha} d^{2} k_{\alpha \perp} d \varpi_{i} d^{2} k_{i \perp}$.

The interaction rate,

$$
\Gamma^{(1, R)}=\sum_{\sigma_{\alpha}, \sigma_{i}} \int d V_{k, R} \frac{\left|\mathcal{A}_{\alpha, i}^{R,(1)}\right|^{2}}{\Delta \tau} n_{F}\left(\varpi_{\alpha}\right)\left[1-n_{F}\left(\varpi_{i}\right)\right],
$$

is obtained by dividing the differential probability by the total proper time $\Delta \tau$, multiplying it by the relevant fermionic thermal factors, and integrating over $d V_{k, R}$.

Following a similar recipe for processes (2) and (3) we can write the total interaction rate, according to Rindler observers, as

$$
\begin{aligned}
& \Gamma^{p \rightarrow n \bar{l}_{\alpha} \nu_{i}, R}=\sum_{j=1}^{3} \Gamma^{(j, R)} \\
= & \frac{G_{F}^{2}\left|U_{\alpha, i}\right|^{2}}{8 a^{2} \pi^{7}} e^{-\pi \Delta m / a} \int_{-\infty}^{\infty} d \varpi_{i} \iint d k_{i}^{x} d k_{\alpha}^{x} \\
\times & \iint d k_{i}^{y} d k_{\alpha}^{y} l_{i} l_{\alpha}\left|K_{1 / 2+i \varpi_{\alpha} / a}\left(l_{\alpha} / a\right)\right|^{2} \\
\times & \left|K_{1 / 2+i\left(\varpi_{\alpha}-\Delta m\right) / a}\left(l_{i} / a\right)\right|^{2} .
\end{aligned}
$$

Now, following the reasoning of Ref. [9] we use Eqs. (6.412) and the definition of the Meijer G-function [Eq. (9.301)] of Ref. [15], along with Eq. (5.6.66) of Ref. [16] to write the total interaction rate according to 
Rindler observers as

$$
\begin{aligned}
& \Gamma{ }^{p \rightarrow n \bar{l}_{\alpha} \nu_{i}, R}=\frac{G_{F}^{2} a^{5}\left|U_{\alpha, i}\right|^{2}}{32 \pi^{7 / 2}} e^{-\pi \Delta m / a} \\
& \times \int_{C_{t}} \frac{d t}{2 \pi i} \int_{C_{s}} \frac{d s}{2 \pi i}|\Gamma(3-s-t+i \Delta m / a)|^{2} \\
& \times \frac{\Gamma(-s) \Gamma(-t) \Gamma(2-t) \Gamma(2-s)}{\Gamma(3-s-t) \Gamma(7 / 2-s-t)}\left[\frac{m_{\alpha}}{a}\right]^{2 t}\left[\frac{m_{i}}{a}\right]^{2 s},
\end{aligned}
$$

which can be seen to be exactly equal to Eq. (49), proving our assertion that both rates coincide.

\section{CONCLUSIONS}

We have discussed the properties of the Unruh thermal bath for neutrinos and in which conditions we can legitimately speak about flavor states. Also, we have shown through an explicit calculation the equality of the decay rates for the inverse $\beta$ decay of protons as calculated by Minkowski and Rindler observers. This is not surprising, being the expected result from the general covariance of quantum field theory, but it explicitly demonstrates the importance of using the appropriate mode expansion (i.e., those that are eigenfunctions of an appropriate timelike isometry) for the neutrino fields, where all calculations are well-defined and it is completely meaningful to speak about particles. Finally, as previously stated, this also shows that there is no incompatibility between the Unruh effect and neutrino mixing, contrary to previous claims.

\section{Acknowledgments}

We are indebted to Vicente Pleitez for insightful comments. G. C. and A. L., G. M., D. V. were fully and partially supported by São Paulo Research Foundation (FAPESP) under Grants 2016/08025-0 and 2014/263078, 2015/22482-2, 2013/12165-4, respectively. G. M. was also partially supported by Conselho Nacional de Desenvolvimento Científico e Tecnológico (CNPq).

\section{Appendix A: Detectors' behavior}

To better understand the behavior of the fermionic detector defined in Sec. II we apply it to an usual thermal bath (at inverse temperature $\beta$ ) of massive neutrinos,

$$
\begin{aligned}
\hat{\rho}_{\beta} & =\bigotimes_{\vec{k}_{i}, \sigma_{i}, i} \frac{1}{1+\exp \left(-\beta \omega_{i}\right)} \\
& \times \sum_{n_{i}=0}^{1} \exp \left(-n_{i} \beta \omega_{i}\right)\left|n_{i} ; \vec{k}_{i}, \sigma_{i}\right\rangle\left\langle n_{i} ; \vec{k}_{i}, \sigma_{i}\right|
\end{aligned}
$$

where $\omega_{i}$ satisfies the usual dispersion relation $\omega_{i}^{2}=$ $\left|\vec{k}_{i}\right|^{2}+m_{i}^{2}$. By using Eq. (20) we calculate the mean excitation rate by absorption of neutrino with mass $m_{i}$ per proper time of an inertial detector with worldline (in $\{t, x, y, z\}$ coordinates $) x_{D}^{\mu}(\tau)=(\tau, 0,0,0)$, giving

$$
\begin{aligned}
\frac{d P_{\mathrm{exc}, i}}{d \tau} & =\pi^{-1}|\lambda|^{2}\left|\left\langle e\left|\hat{m}_{0}(0)\right| g\right\rangle\right|^{2} \int_{m_{i}}^{\infty} d \omega \delta(\omega-\Delta E) \\
& \times \frac{\omega \sqrt{\omega^{2}-m_{i}^{2}}}{e^{\beta \omega}+1}
\end{aligned}
$$

which, disregarding the phase-space volume factor, is basically proportional to the mean number of particles with energy $\Delta E$ as it should be. We also note that this detector satisfies the detailed balance condition, relating excitation and absorption rates [7]. Moreover, the excitation rate in this case is also proportional to the local neutrino density calculated from Eq. (27), which, in this case is

$$
\left\langle\hat{n}_{i, D}\right\rangle_{\hat{\rho}_{\beta}}=\frac{1}{\pi^{2}} \int_{m_{i}}^{\infty} d \omega_{i} \frac{\omega_{i} \sqrt{\omega_{i}^{2}-m_{i}^{2}}}{e^{\beta \omega_{i}}+1}
$$

[1] W. G. Unruh, "Notes on black hole evaporation," Phys. Rev. D 14, 870 (1976).

[2] J. J. Bisognano and E. H. Wichmann, "On the duality condition for quantum fields," J. Math. Phys. 17, 303 (1976).

[3] G. L. Sewell, "Quantum fields on manifolds: PCT and gravitationally induced thermal states," Ann. Phys. 141, 201 (1982).

[4] G. W. Gibbons and M. J. Perry "Black holes in thermal equilibrium," Phys. Rev. D 16, 985 (1976)

[5] G. W. Gibbons and M. J. Perry, "Black holes and thermal Green functions," Proc. Roy. Soc. London A 358 (1978)

[6] D. V. Ahluwalia, L. Labun and G. Torrieri, "Neutrino mixing in accelerated proton decays," Eur. Phys. J. A 52, 189 (2016).

[7] L. C. B. Crispino, A. Higuchi, and G. E. A. Matsas, "The
Unruh effect and its applications," Rev. Mod. Phys. 80, 787 (2008).

[8] G. Cozzella, A. G. S. Landulfo, G. E. A. Matsas and D. A. T. Vanzella, "Proposal of observing the Unruh effect with classical electrodynamics," Phys. Rev. Lett. 118, 161102 (2017).

[9] H. Suzuki and K. Yamada, "Analytic evaluation of the decay rate for an accelerated proton," Phys. Rev. D 67, 065002 (2003).

[10] F. Kiaka, A. R. H. Smith, M. Ahmadi and A. Dragan, "Massive Unruh particles cannot be directly observed," arXiv:1711.05179 [quant-ph]

[11] J. Castiñeiras, L. C. B. Crispino, G. E. A. Matsas and D. A. T. Vanzella, "Free massive particles with total energy $E<m c^{2}$ in curved spacetimes," Phys. Rev. D 65, 104019

$[12]$ D. A. T. Vanzella and G. E. A. Matsas, "Decay of accel- 
erated protons and the existence of the Fulling-DaviesUnruh effect," Phys. Rev. Lett. 87, 151301 (2001).

[13] B. Pontecorvo, Sov. Phys. JETP 26, 984 (1968)

[14] M. Blasone, G. Lambiase and G. G. Luciano, "Nonthermal signature of the Unruh effect in field mixing," Phys. Rev. D 96, 025023 (2017).

[15] I. S. Gradshteyn and I. M. Ryzhik, Table of Integrals, Series and Products (Academic Press, New York, 1980).

[16] H. Bateman, Higher transcendental functions, Vol. I (McGraw-Hill, New York, 1953).

[17] C. Giunti \& C. W. Kim, Fundamentals of neutrino physics and astrophysics (Oxford University Press, Oxford, 2007).

[18] C. Giunti, C. W. Kim \& U. W. Lee, "Remarks on the weak states of neutrinos" Phys. Rev. D 45, 7, 2414 (1992)

[19] S. Bilenky, Introduction to the physics of massive and mixed neutrinos Springer-Verlag, Berlin Heidelberg, 2010

[20] G. Cozzella \& C. Giunti; In preparation

[21] B. Kayser, "On the quantum mechanics of neutrino os- cillation," Phys. Rev. D 24, 110 (1981).

[22] The flavor number operator constructed from Eq. (C10) of Ref. [14], gives, e.g., a non-zero expectation value in the the standard inertial vacuum $\left|0_{\mathrm{M}}\right\rangle$.

[23] This statement must be made compatible with situations where it seems sensible to speak of flavor neutrino particles, such as neutrino oscillation experiments. In most of these experimental situations, the momenta involved are much larger than the neutrino masses $m_{i}$ and it is useful to define the states given by Eq. (32), coming from the limit of weak-processes states [17] where there is not enough precision to determine the different neutrino masses. It is also tactically assumed enough uncertainty in the momenta determination in such a way that the neutrinos are sufficiently localized to allow oscillations to be seen [21]. A more physically complete (and technically challenging) picture is obtained by working with wave-packets instead of plane waves. 